
\title{
Symmetry breaking and functional incompleteness in biological systems
}

\author{
Andrej Korenić \\ The Center for Laser Microscopy \\ Institute for Physiology and Biochemistry \\ Faculty of Biology, University of Belgrade \\ Slobodan Perović \\ Department of Philosophy \\ Faculty of Philosophy \\ University of Belgrade \\ Milan Ćirković \\ Astronomical Observatory Belgrade \\ Paul-Antoine Miquel \\ Laboratoire Erraphis \\ Universté de Toulouse 2
}

\begin{abstract}
Symmetry-based explanations using symmetry breaking (SB) as the key explanatory tool have complemented and replaced traditional causal explanations in various domains of physics. The process of spontaneous SB is now a mainstay of contemporary explanatory accounts of large chunks of condensed-matter physics, quantum field theory, nonlinear dynamics, cosmology, and other disciplines. A wide range of empirical research into various phenomena related to symmetries and SB across biological scales has accumulated as well. Led by these results, we identify and explain some common features of the emergence, propagation, and cascading of SB-induced layers across the biosphere. These features are predicated on the thermodynamic openness and intrinsic functional incompleteness of the systems at stake and have not been systematically analyzed from a general philosophical and methodological perspective. We also consider possible continuity of SB across the physical and biological world and discuss the connection between Darwinism and SB-based analysis of the biosphere and its history.
\end{abstract}

Keywords: Symmetry breaking; Phase-transitions; Open systems; Homochirality; Biosphere. 


\section{Introduction}

We are all intuitively aware of the bilateral or mirror symmetry of our bodies on the left and right sides on the (mid)sagittal plane. Two other planes, transverse (horizontal) and coronal (frontal), have asymmetric attributes along the craniocaudal (anteroposterior) and dorsoventral axes. By the same token, biologists studying unicellular organisms have located various polarities that affect both their morphology and functionality, while those studying the structure of tissues have identified asymmetric traits. Yet attempts to develop a general idea of the role of symmetry and symmetry breaking (SB) in biology have been ignored or dismissed as forced attempts to return to a pre-Darwinian way of thinking. It is even more difficult to develop such an idea at the molecular level.

In physics, however, symmetry and symmetry breaking have been used as a conceptual tool to identify subatomic particles and their properties for many decades. In fact, the convergence of theory describing particle properties and group theory is the foundation of the Standard Model of particle physics. The same convergence of constituent properties and their symmetries explained by group theory facilitated the understanding of solid-state physical phenomena, and, consequently, of materials science (e.g. Elliott and Dawber 1985).

The inherent link between physical conservation laws and group theory was first spelled out by Emmy Noether and has since been elaborated on by such giants of modern physics as Eugene Wigner, Kenneth Wilson, Chen-Ning Yang, Steven Weinberg, and others. That sort of account of micro-physical properties has been an extremely fruitful pursuit in mathematical physics, but it has also been used across the field to explain a diverse range of physical phenomena. The majority of the great discoveries in the last half century, like graphene or Higgs' boson, would have been inconceivable without understanding the role of symmetry in physics. 
Although asymmetric structures of living matter are obvious at the scale of organisms, studying them by attempting to comprehensively connect the roles they play across biological levels is not yet part of a detailed research program. However, a wide range of empirical research into various phenomena related to symmetries and symmetry breaking across biological scales has accumulated over the last two decades. These diverse studies utilize various notions of symmetry breaking as both a theoretical and an explanatory tool. This invites exploration of possible common, systemic traits, as well as links with symmetry breaking in the microphysical world.

We start this paper with a succinct characterization of symmetry-based explanations in physics as they complement and, in many instances, replace the traditional causal explanatory framework. Next, we briefly turn to symmetry breaking (SB) in physical systems and explain the basic concepts of open systems and phase changes crucial to applying the concept of SB in biology. Then, based on this theoretical framework, we identify the basic structure of SB layers in the biosphere, starting with a detailed account of homochirality; we discuss the propagation of SB layers and explain their cascading across the biosphere's levels. Finally, we conjecturally consider the possible continuity of SB across the physical and biological world and discuss the connection between Darwinism and SB-based analysis of the biosphere.

\section{Symmetries and symmetry breaking in physics and in}

\section{biology: a general framework}

\subsection{Causal and symmetry-based explanations in physics}

To show how explanations based on symmetries and symmetry breaking compare to the traditional explanations of physical phenomena in their causal determination, we begin with an example. The tentative relationship between these two kinds of explanations and the 
explanatory gain when the former is used are already apparent in the simple case of a planet revolving around the Sun (as accounted for in classical physics, in particular, celestial mechanics).

The relevant physical parameters in this case are the position and velocity of the planet. Position and velocity are measurable physical properties; their measurement creates a set of values which describe a state in a physical system. In classical mechanics, the phase space consists of all possible states, i.e. values of position and velocity. In the case of an elliptic trajectory, the position and velocity of the planet vary continuously, but the surface of circle sectors swept out by the planet turning around the Sun will not change within equal intervals of time, expressing the conservation of mechanical energy (Kepler's second law) - an invariant quantity corresponding to symmetry (Figure 1).

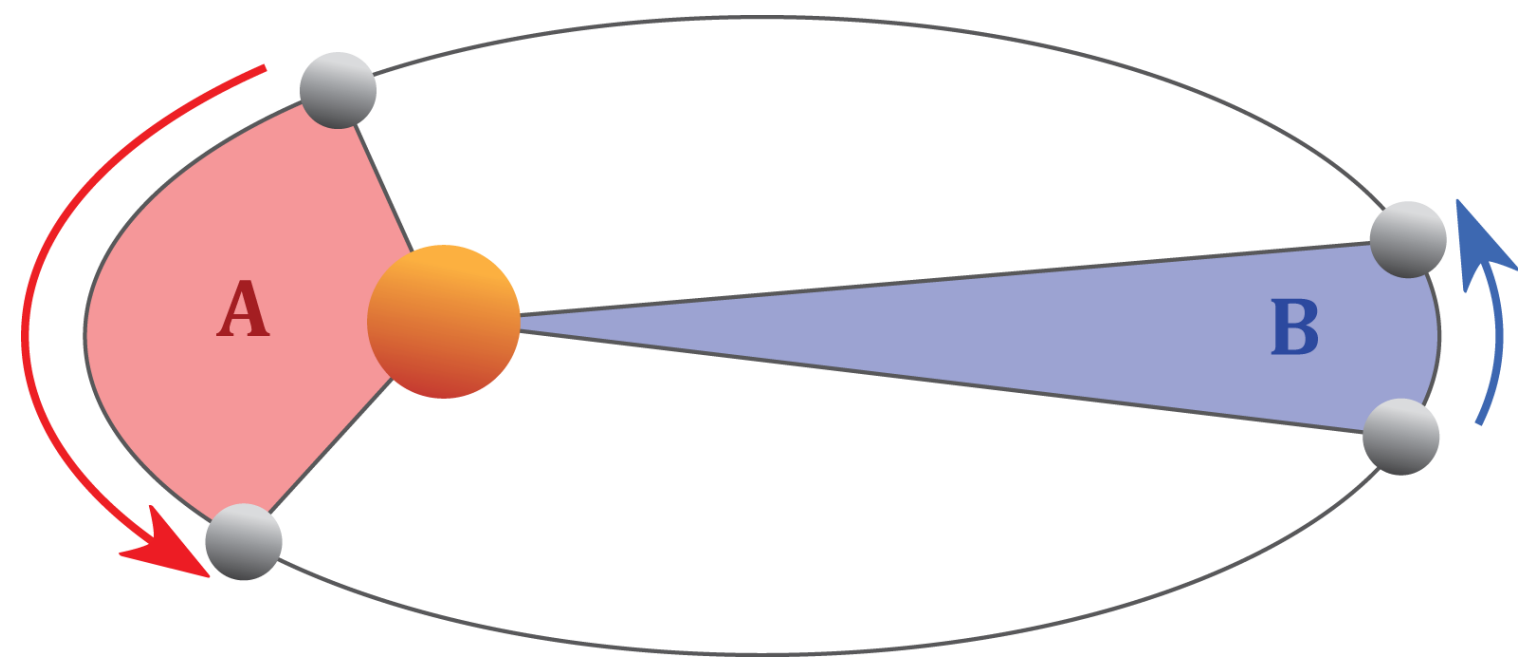

Figure 1. A simple example of symmetry invariance in physics. The imaginary line joining the center of the planet to the center of the Sun sweeps out the same circle sector in equal intervals of time. The arrows depict the direction of rotation and the distance the planet travels in a given interval of time. 
A physical state is a theoretical posit; although observable, it is not merely an empirical posit. It is, in a sense, nothing but a value expressed by a variable, or by a function in a differential equation. Thus, an observed event cannot be simply reduced to a physical state ${ }^{1}$.

Now, should causal relations describe the observed anteriority of an event A to another event $B$ (Figure 1)? And should we say that the Sun is the cause of the planet's movement? The answer is not simple because, at the same time, the planet is interacting with the Sun, thanks to the principle of equality between action and reaction. Strictly speaking, all we know is a causal correlation, or a differential cause, not simply a cause. However, in a reference system (that is, where one thing is always relative to another), we observe that the planet is moving around the Sun, because of the increase and the decrease in the velocity of the planet and its distance from the Sun. In this case, we can say that an effect (the movement of the planet) cannot occur before its differential cause (the interaction with the Sun). As such, the causality principle works in all classical theories in physics. And it leads to the representation of time as an arrow coming from past and going to the future. ${ }^{2}$

In contrast to such a causal explanatory account, the system can be deemed determinate if there is some kind of physical hierarchical dependency relationship between the structure and the states of a local system. A change of states in the system will never affect the structure in

\footnotetext{
${ }^{1}$ Moreover, physical states and observed events may be treated as synonymous in classical mechanics, but certainly not in quantum physics.

${ }^{2}$ Even Minkowsky's light-cone is compatible with such a representation. If the Sun were to magically vanish, the Earth would have revolved around nothing for further 8.3 minutes, before propagation of causal influence would cause it to continue its inertial motion along the tangent. Adding relativity constrains the spatiotemporal volume of possible observations, but our interpretation of the physical state easily accommodates such constraint.
} 
such a way that there is an asymmetrical relationship between states and structure. Expressed in classical physics as a symmetry group, this configuration encodes symmetry features as a set of transformations and their combinations that leave the object (structure) as unchanged (conserved).

Symmetries have a conservative nature because they are transformations that can be inverted (Fraassen, 1989; Longo et al., 2012). For instance, in our example and thanks to the system's symmetry invariance, when the distance to the Sun increases, the velocity of the planet decreases, so the mechanical energy always remains the same. More technically, symmetries by time-translation are associated with energy-conservation, as initially suggested by Noether's theorem. Strictly speaking, such a compositional, hierarchical, and mediated relation between states and structure cannot be treated causally, because the space-time path of the elements coexists with the space-time path of the structure. The structure will not exist before the states and vice versa.

The epistemic and explanatory gain in the latter account is that we can predict a final state of the system from initial conditions, if we know what relevant symmetries the change of the system is invariant to - and not only if we know differential causes. The prediction is based on the theoretical and mathematical structure at work in a natural system. This is not simply an empirically based causal account in the sense of identifying differential causes (as e.g. David Hume believed).

\subsection{Phase transitions in thermodynamically open systems and symmetry breaking}

Biological systems are thermodynamically open systems characterized by critical transitions and phase changes. These characteristics play a crucial role in symmetry breaking, 
so we need to explain them briefly to explicate the notion and the explanatory role of symmetry breaking in biology.

Thanks to the aforementioned link between symmetry groups and physical structure, one class of theoretical physical systems can be characterized as closed and conservative (Nicolis and Prigogine, 1989). "Closure" is coextensive with completeness: put otherwise, there is no physical state that cannot be fully determined by its relationship to the physical structure thanks to the connection between the symmetry groups and the principles of conservation. In our example of a planet orbiting the Sun, closure is expressed by the use of the Hamiltonian operator "H" ("q" and "p" are positions and velocities):

$$
\begin{gathered}
\frac{\partial H}{\partial p}=\frac{d q}{d t} \\
-\frac{\partial H}{\partial q}=\frac{d p}{d t}
\end{gathered}
$$

"Conservation" means that a change of a state in the system will never affect the structure when every change of the state is at the same time determined by the structure, thanks to the condition given in equation (1). To put it slightly differently, the relationship between a state and the structure is not adequately described simply as a causal one, because the structure cannot be treated as an effect of any change; rather, it "picks out" the condition for every change:

$$
\frac{\mathrm{dH}}{\mathrm{dt}}=0
$$

Therefore, the necessary condition for the conservation laws to obtain in a system is the system's closure, that is, its completeness. It should be noted that the presence of conservative principles constitutes the difference between a law of nature, like "every body is heavy", and a universal proposition, like "every crow is black", without any abstruse or artificial 
speculation on counterfactual conditionals. The statement "every crow is black" is not integrated into a compositional structure expressed by the use of symmetry groups and conservative principles; thus, it is not a law of nature. This point was emphasized by French physicist Pierre Duhem (1902) and Austrian physicist Ernst Mach at the beginning of the $20^{\text {th }}$ century but has been lost in contemporary philosophy of science, perhaps because of its focus on the semantic and logical analysis of scientific theories.

In this sense, completeness characterizes any closed system, but it is certainly not an essential property of the physical world in general. In fact, advances across the scientific disciplines, from cosmology to computer science to geosciences, have led to the realization that the vast majority of physical systems are open systems. It is not surprising, then, that a distinction was made between "closed" and "open" thermodynamic systems (von Bertalanffy's individual growth model; Von Bertalanffy and Sutherland, 1974) before this distinction was developed and expressed by theoretical physicists (Nicolis and Prigogine, 1977).

The concept of a closed system in physics is simply a strong idealization, nothing but the expression of the fact that macroscopic thermodynamics cannot be fully recovered by Statistical Mechanics without the use of infinite idealizations because phase transitions, a widespread and basic physical phenomenon, can occur only in open thermodynamic systems, in either a weak (energy exchanges) or a strong (matter and energy exchanges) use of this term. Examples are ubiquitous and diverse and include phenomena like changes from solids to fluids and vice versa (e.g. in the case of water and ice), dissolution and crystallization (e.g. carbohydrates or amino acids), or the racemization of amino acids (Figure 2).

Such phase transitions essentially "break" the usual framework of Statistical Mechanics. The difficulty of accounting for phase transitions can be overcome by taking "thermodynamic limit" into account. For an infinite idealized system of particles occupying an infinite volume, 
the partition function $\mathrm{Z}$ characterizing its physical structure can harbor singularities. Yet, as pointed out by Callender (2001), phase transitions occur in finite systems in nature: "Phase transitions — as understood by statistical mechanics — can only occur in infinite systems, yet the phenomena that we are trying to explain clearly occur in finite systems" (2001, p. 549).

Such physical systems are, in effect, incomplete because the characterization of the states of the system does not simply depend on the system's internal physical structure (Nicolis and Prigogine, 1989), and singularities that are not directly analytically solved can appear in it. These physical systems also depend on boundary conditions: a flow equation characterizing the relation between the systems and their boundary conditions can be drawn, but it exhibits singularities at critical points, and such systems will not conserve their initial structures.

In other words, these systems exhibit symmetry breaking $(S B)$ at the critical point of phase transition. Unlike the classical systems in physics, SB cannot be explained by an inherent relationship between appropriate symmetry groups and conservation principles. Instead, at the critical point, SB exhibits new global properties expressed by order parameters, like the loss of entropy, clustering distribution indices, or infinite correlation length. ${ }^{3}$

\footnotetext{
${ }^{3}$ It should be noted that we could characterize phase changes, such as the phases water takes up, as symmetry changes rather than symmetry breaks since the system can turn back to the previous change with a simple rotation (Montévil et al. 2016). Yet we opt to characterize this change as SB because the characteristics of the phase changes we describe here contrast with those of classical systems. This does not substantially contradict the characterization of Montévil et al. 2016 - only the focus is different. In biological systems, as we will see, the system that undergoes SB cannot go back (while maintaining the biological function and coherence of a living unit) to the previous phase by simple transformation.
} 


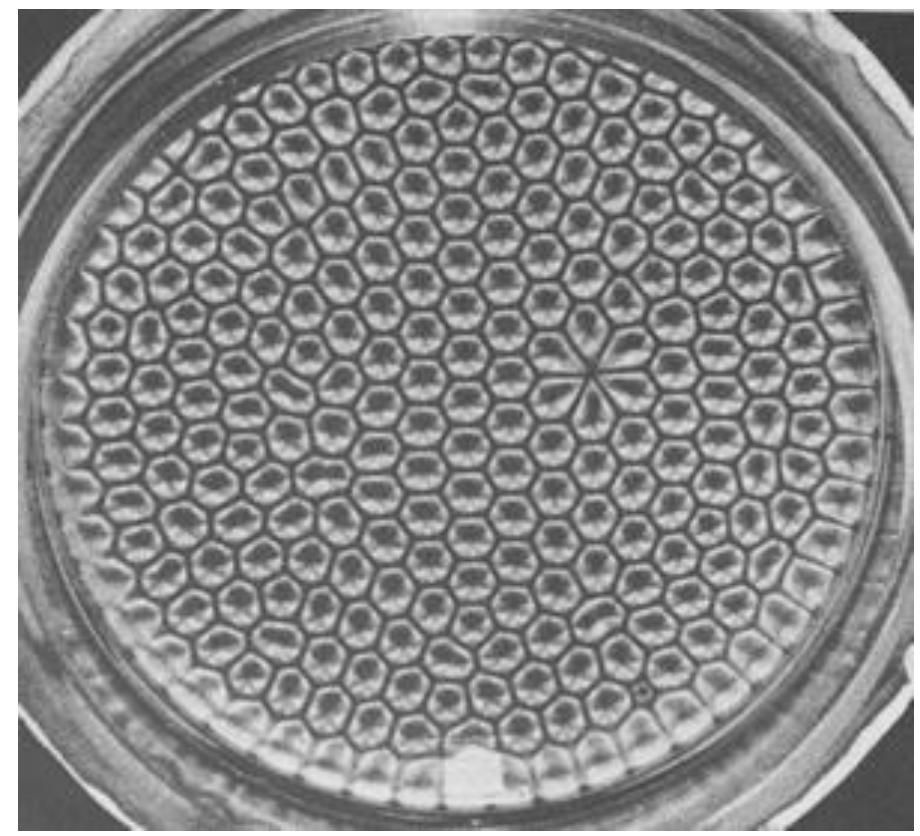

Figure 2. Convection patterns formed during phase-change: Rayleigh-Bénard (thermal) convection occurs in a horizontal layer of fluid heated from below. A regular pattern of convection cells is known as Bénard cells. Notice the small "glitch" in the pattern where cells are not hexagonal - a tiny dent in the copper plate can change the pattern.

Thus, the dynamics of the system are not fully explained by the initial structure - that structure should be invariant under any transformation with respect to the symmetries defined by an appropriate symmetry group. In fact, the structure of the system at the critical point is a result of the system's operations, not the structure itself. This sort of physical change can only be accounted for by some kind of fixed point equation using the sophisticated technique of renormalization groups. As explained by Maris and Kadanoff (1978), "renormalization” (R) simply means "recursion relations" by means of which critical exponents can be calculated in a space of mappings:

$$
{ }^{\prime} \mathrm{R}=\phi \mathrm{R}
$$

Note that this space of mappings (Collet and Eckmann, 2009; Lesne et al., 2014) is not the phase space used for classical theories in physics as this was sketched above. As we have pointed out, in this phase space, the structure of the system analyzed is nothing but the result of its operations. 
Following Montévil and Mossio, the new global properties that emerge over time in critical systems can be called constraints (Montévil and Mossio, 2015). Constraints are expressed by recursive fixed-point equations that permit us to calculate critical exponents that are simply the signature of the emergence of new global properties in a local system. Constraints are neither laws, nor universal properties. They only emerge in open physical systems submitted to specific boundary conditions. They cannot be determined by classical symmetry groups in a classical phase space. Constraints are not included in the laws of conservation, because they are context- and timescale-dependent. We cannot pretend that they obey the principle of covariance at work in classical physics, wherein: "[t]he equation is covariant if it is either true for all the frame of reference or for none" (Fraassen, 1989, p. $281)^{4}$

\subsection{Symmetry breaking in biological open systems}

Now that we have defined the basic notions we need to understand SB in biology, we can look at some examples. The formation of homochirality in amino acids and carbohydrates, to which we turn shortly, is the basic SB relevant to the structure of the living world. For example, proteins are biological polymers with only $L$ (no $D$ ) constituents of amino acids. Louis Pasteur used the homochirality of biomolecules as the defining property of life - this deserves more than a historical footnote, given the tremendous difficulties involved in the foundational project of defining life (Cleland, 2012; Cleland and Chyba, 2002). Pasteur's early, and at the time radical, idea attests to the relevance of SB to biology on the molecular level and above.

\footnotetext{
${ }^{4}$ As such, they can be defined as entities that control or regulate the activity of other entities (Montévil et al., 2016).
} 
To return to the topic at hand, however, in this section, we make some general introductory remarks about SB in biology within the conceptual framework outlined in previous sections. We then explain the details of homochirality and identify the structure of various other SB layers in the biosphere.

In general, we argue that the major difference between physical and biological open systems is that in the latter, we face extended criticality, i.e. protracted and intertwined multiple critical phase changes (Balian, 1998). A biological system is continuously generating new constraints through a continuous flow of symmetry breaking, causing its space of constraints to appear open-ended (Kauffman, 2000; Longo et al., 2012; Longo and Montévil, 2014, 2011; Montévil et al., 2016). In other words, the space of constraints is not generic; neither, accordingly, is any specificity of evolutionary and ontogenic trajectories predictable.

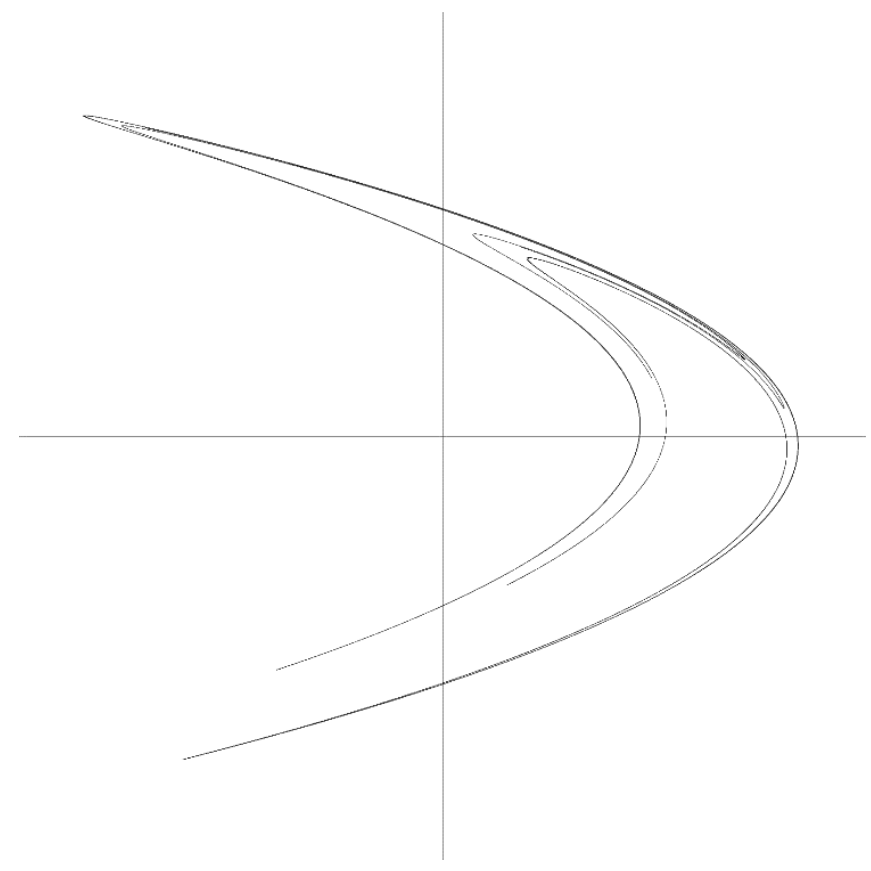

Figure 3. Hénon's attractor, as an example of a strange attractor in dynamical systems. Hénon's map is a product of relatively simple non-linear $2 \mathrm{D}$ transformations, but its long-term behavior during iterations produces extraordinarily complex phenomena (which we might call 'patterns').

Unpredictability in dynamical physical systems is associated with sensibility to initial conditions, with topologic transitivity, and with density of periodic orbits. In contrast, in biology, unpredictability is associated with the fact that pathways and trajectories always 
occur in an open-ended space of constraints, and this space continuously regenerates itself (Figure 3). Thus, a biological system appears as a kind of autonomous device (Kauffman, 2000) that is always regenerating and propagating itself through one or through multiple thermodynamic virtuous cycles.

The first consequence of this regeneration is that such a system is incomplete because its structure and symmetries are continuously modified by the pathway and trajectories of the system itself. In other words, the synchronic structure of an extended critical system is continuously modified by its dynamics. To sum up, there are three interrelated aspects of functional incompleteness. First, the characterization of relevant systems does not simply depend on their internal physical structure; it depends on singularities (phase changes) that cannot be solved analytically and are highly sensitive to changes in initial conditions. Second, the systems regenerate themselves through the self-propagation of specific thermodynamic cycles; this way, they retain SB cycles as the preferred stable modifications. Third, due to these previous two characteristics, the transformation of such systems takes a unidirectional route; unlike certain simple systems (e.g. liquid water and ice by simple rotation), they cannot change back to the previous phase after SB by a simple symmetry transformation.

The second, related consequence is that robustness is a secondary property of biological systems, not an essential one, if we define robustness as an "organizational closure" (Montévil and Mossio, 2015), or more generally as "stability with variation". A biological system is structurally unstable, and organizational closure is only a consequence of various interactions between multiple biological systems. 


\section{Symmetry breaking and its propagation in living systems}

In this section, we turn to concrete examples in the living world to show how the preceding theoretical points constitute a general and surprisingly simple pattern characterizing the cycle of life. One example that stands out among many is the Central Dogma of molecular biology, rightly regarded a classic case of SB by Koonin (2015). Yet since a detailed review of the scope of SB in living systems would be vast, in our exemplary discussion, we focused on the homochirality of biomolecules, first, because of its historical importance, i.e., its probable role in the origin of life, and second, because of the very transparent main features of the SB cycle that transpire in it. Section 4.1 contains other key examples that emerged in the cycle of the evolution of life.

We focus on how the propagation of symmetry breaking and constraints might be related to specific biological devices at the molecular and developmental level and at the level of the biosphere (exaptation, Gould and Vrba 1982). We define "plasticity" as a propagative second order trait through which new constraints can be generated from old ones. Finally, we specify the link between plasticity, adaptability, and robustness when the development of organisms is performed in a space of constraints that is never completely closed on itself.

\subsection{Homochirality of biomolecules}

As noted in the previous section, the formation of homochirality in amino acids and carbohydrates appears to be the initial symmetry breaking (SB) propagated in the living world since its inception. Although amino acids and carbohydrates can occur in $(L)$ - and $(D)$-forms (stereoisomers), life on Earth almost exclusively uses only $(L)$-amino acids and $(D)$ - 
carbohydrates. ${ }^{5}$ This seems to be a simple case of a phase change (Blackmond and Klussmann, 2007) exhibiting SB.

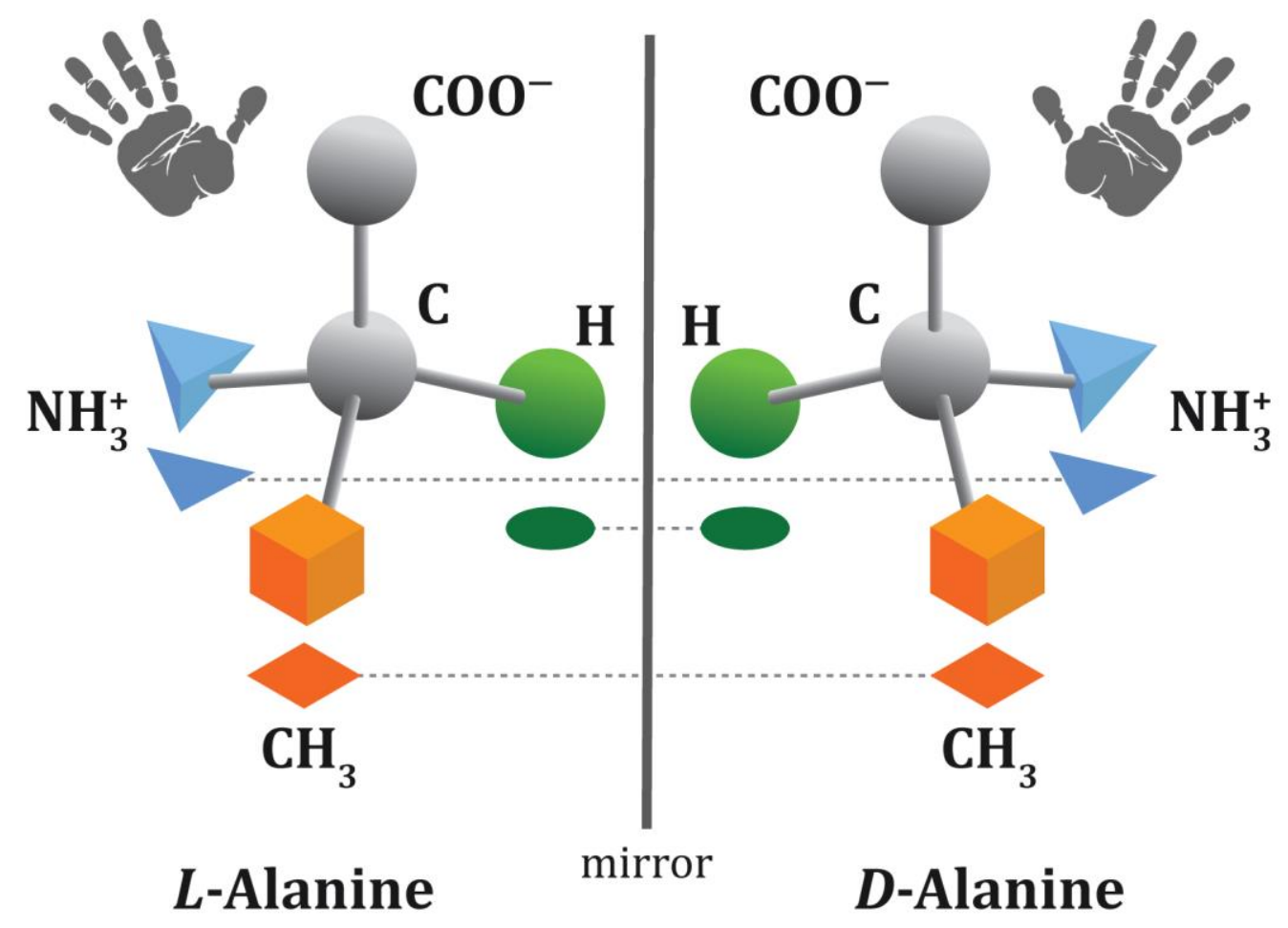

Figure 4. Schematic representation of the mirror symmetry of two enantiomers L- and D-Alanine with the alpha carbon as a chiral center of the molecule, carboxylic acid group $\left(\mathrm{COO}^{-}\right)$on the top, and side chain $\left(\mathrm{CH}_{3}\right)$ on the bottom (referring to a Fischer projection). If the amine group $\left(\mathrm{NH}_{3}{ }^{+}\right)$is on the left side of the carbon chain, the molecule is $\mathrm{L}$ oriented, and if it is on the right, the molecule is D oriented. Geometric shapes below the models of the molecules depict projections of three distinct constituents (three vertices of the tetrahedron) on the flat surface.

In the mixture of enantiomers, pairs of molecules that are non-superimposable mirror each other (Figure 4). Their thermodynamic and physical properties (other than light polarization and, more importantly perhaps, their energy efficiency - see below) are invariant under the difference in chirality and are therefore used as a label: $L$ and $D$ enantiomers (like 0/1 in

\footnotetext{
${ }^{5}$ Yet the terms left and right are part of the convention, like, for example, the right-hand (thumb) rule is in electromagnetism. Our hands preserve their property of chirality even when we cross them (with open palms facing us).
} 
binary code or head/tail of a coin; see Figure 5). If we consider the racemization process of $N$ molecules of $L$ and $D$ enantiomers, a microstate $x$ is one particular picture of $N$ enantiomers from a set of all possible $(w)$ microstates $\left(M_{x}\right)$ with associated probabilities $\left(P_{x}\right)$ :

$$
\begin{gathered}
M_{X}=\left\{m_{1}, m_{2}, \ldots, m_{i}, \ldots, m_{w}\right\} \\
P_{X}=\left\{p_{1}, p_{2}, \ldots, p_{i}, \ldots, p_{w}\right\}
\end{gathered}
$$

where $w=2^{N}$ (number of "ways"). Given that $p_{(L)}=p_{(D)}=0.5$ (i.e. invariant under the difference in chirality) and $p_{i}=1 / w$, the maximum entropy $(L)$, following Gibbs-Shannon expression,

$$
S=-\sum_{i=1}^{w} p_{i} \ln p_{i}
$$

becomes $L=\ln w=\ln 2^{N}$. If the change of entropy is expressed over the change of information $\Delta S=\Delta L-\Delta I$, then the "revised information theory" predicts a tendency towards the highest possible dynamic symmetry at higher temperatures ("information registration") and the highest possible static symmetry ("information loss") at lower temperatures. Put otherwise, although the static structure can be any of the $w$ microstates, when e.g. thermodynamic temperature is reduced, entropy can be increased through the spontaneous separation of different components (for a detailed mathematical explanation, see Lin 2001).

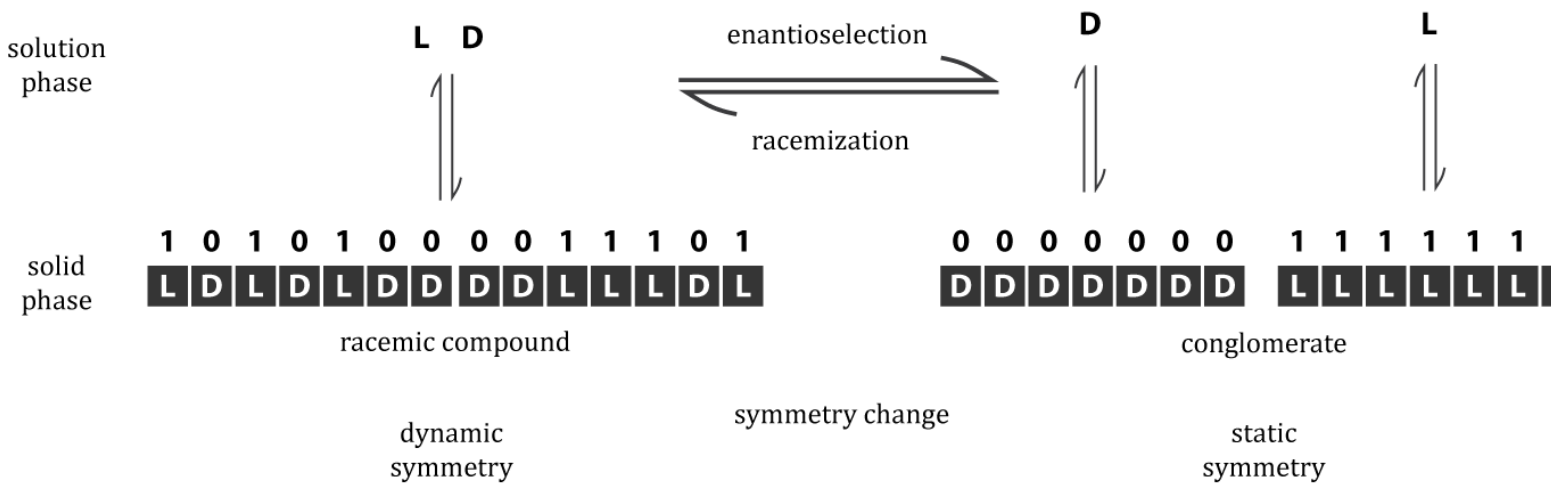

Figure 5. Depiction of equilibrium between solid phase and solution phase and between (any) processes of the 
enantioselection and racemization of amino acids (adapted from Blackmond, 2010 and Lin, 2001). Boxed letters L and D represent solid phase enantiomeric molecules either as racemic compounds or as conglomerates; letters without boxes represent solution phase molecules in equilibrium with solid phase. The chirality of the two different enantiomers L and D can be used as a label, as relevant thermodynamic and physical properties other than light polarization are indistinguishable (or symmetric), much like two sides of a coin or $0 / 1$ (given that $\mathrm{p}_{(\mathrm{L})}=\mathrm{p}_{(\mathrm{D})}=0.5$ ). Thus, symmetry breaking can be characterized as a permanent shift between dynamic and static symmetry (in either direction).

In a nutshell, hypotheses about the origin of the homochirality ('enantioselection direction' in Figure 5) of amino acids and carbohydrates, more specifically, their imbalance that is traditionally measured in terms of the enantiomeric excess (ee), can be divided into two broad categories: hypotheses on abiotic and biotic origins (Aruna, 2006; Meierhenrich, 2008).

\subsection{Abiotic origin of homochirality of amino acids}

The number of described mechanisms according to which asymmetry is a result of environmental conditions has grown in the past decade. The diversity of abiotic mechanisms increases the probability that any small (local) asymmetry created in an abiotic way could be amplified by an intervening biotic process, thus increasing the cell's fitness (i.e. error-free translation process resulting in the stability of more complex proteins).

\subsubsection{Autocatalytic reaction coupled with polymerization}

After a long life as a purely theoretical concept originally described by Frank (1953), an autocatalytic reaction mechanism for the evolution of homochirality was confirmed in Soai's (1995) reaction. In this reaction, autocatalysis is coupled with a form of "mutual antagonism". Frank's concept was further elucidated by Blackmond/Brown's kinetic model as an inactive heterochiral dimer catalyst. As the model shows, a small nudge from the reaction product (catalyst) or other chiral molecules is needed to constantly and irreversibly direct the reaction toward enantioenrichment (Blackmond, 2010).

Furthermore, according to the "polymerization on the rocks" theory (Orgel 1998), minerals provide Earth's most chirally biased environments, i.e. environments promoting "mineralinduced selection, concentration, and organization of prebiotic molecules into viable 
macromolecules" (Hazen, 2006). Not only do mineral-molecule interactions have a possible role to play in the origin of biochemical homochirality, but mineral-induced (i.e. nonenzymatic) polymerization ${ }^{6}$ yields mole fractions of homochiral oligopeptide sequences larger than those calculated for a theoretical, random polymerization process (binomial distribution) (Hitz and Luisi, 2003). This phenomenon has also been demonstrated for an air-water interface and in the presence of liposomes (for comprehensive reviews, see Pressman, Blanco, and Chen 2015; Hazen and Sverjensky 2010). Furthermore, polymerization of same-handed molecules might be favored by chirality-induced spin polarization which places less repulsive symmetry constraints than opposite handedness (Kumar et al., 2017).

\subsubsection{Phase change models}

It has been suggested that the first enantiomeric imbalance ${ }^{7}$ might have been brought to Earth by the fractionation of organic space dust via sublimation (Blackmond and Klussmann, 2007). Other possible mechanisms include chiral amnesia and crystal engineering phase behavior models; the former is based on a combination of the "Eve Crystal" model of crystallization for conglomerates and solution-phase racemization, and the latter is based on the thermodynamics of chiral compounds when they form relatively insoluble racemic compounds with small, achiral molecules incorporated in the crystal structure. Chiral amnesia results in the conversion of one enantiomer to the other, whereas crystal engineering merely partitions the existing molecules between phases (Blackmond, 2010).

Moreover, "further amplification [of such initial enantiomeric imbalance] could take place in terrestrial aqueous pools, where solution-solid equilibrium might be established over time

\footnotetext{
${ }^{6}$ Carbonyl diimidazole (CDI) and 1-ethyl-3-(3-dimethylaminopropyl) carbodiimide (EDAC) are relevant to prebiotic chemistry ("polymerization on the rocks" protocol).

${ }^{7}$ In these cases, we assume $0 \% e e$, in other words, equal concentrations of enantiomers.
} 
through cycles of rainfall and evaporation, leading to highly enantioenriched sublimates or solutions, respectively, for some amino acids". Therefore, gas and solid phase transitions both provide "a plausible mechanism for partitioning or fractionation of enantiomer composition ${ }^{8}$ through amplification of enantiomeric excess based on amino acid phase behavior" (Blackmond and Klussmann, 2007; Viedma, 2005).

Either way, because of its persistence and amplification, especially during the initial stage of phase change, possibly due to adequate temperature, homochirality is the basic building layer of the life-cycle of the biosphere and, thus, represents a turning-point in the evolution of life. Theoretically speaking, in this case, we are dealing with the same singularities as in the phase changes described earlier, but grasping this phase change requires experimentation and modeling (Blackmond and Klussmann, 2007) as there is no neat overarching theoretical formal account.

\subsubsection{Photochemical model of enantioselection}

The Parity Violation theories of the origin of homochirality are motivated by the spirit of recent world-wide efforts in physics to elucidate charge-parity (CP) symmetry violation, matter-antimatter asymmetry, and the origin of dark matter. The theories are plausible, as an enantiomeric excess could be extraterrestrial in origin.

Now, although the energy gap between enantiomers produced by parity violation is currently not detectable by any technique (Myrgorodska et al., 2017), we may not need such a technique, as the "one-handedness" of biomolecules could be accomplished by an asymmetrical astrophysical process, namely, the interaction of circularly polarized ultraviolet radiation (UV-CPL) with amino acids/carbohydrates (Vester-Ulbricht processes; De

\footnotetext{
${ }^{8}$ This also includes the differentiation of racemic compounds from conglomerates.
} 
Marcellus et al. 2011; Dreiling and Gay 2014), thus making one enantiomer more stable than the other (Yamagata processes; for comprehensive review, see Meierhenrich 2008).

When we summarize many sources of evidence, including the enantioselective analysis of meteorites, the detection of circularly polarized electromagnetic radiation in star-forming regions, and laboratory simulations of interstellar ices, we clearly see the correlation between laboratory simulations of photochemically induced $e e$ values and cometary composition, providing support for photochirogenesis. We also recognize that stereo-dictating elements of nucleic acids and proteins, sugars, and amino acids might have had a common precursor: chiral aldehydes (glyceraldehyde) (Myrgorodska et al., 2017).

\subsection{Biotic origin of homochirality of amino acids}

Both $(D)$ - and $(L)$-amino acids (DAAs and LAAs, respectively) are synthesized by racemase enzymes and can be found at high concentrations in cells (for more references, see Bhatt, Soni, and Sharma 2016). Since the presence of DAAs can hamper the protein translational process, several cellular mechanisms are dedicated to protecting the process against possible errors in protein synthesis and in its secondary structure. These mechanisms include two types of enzymes: D-amino acid oxidase (dehydrogenase in bacteria and plants) (Wang et al., 2012) and D-Tyr-tRNA deacylase (or D-aa-tRNA deacylase, DTD) (Bhatt et al., 2016; Hussain et al., 2006). Another mechanism is the ability of the ribosome's peptidyltransferase center to discriminate the chirality of amino acids (Englander et al., 2015).

D-amino acid oxidase is known to catalyze the oxidative deamination of neutral and basic DAAs and is responsible for chiral inversion. This "may be a remnant of evolution that favored chiral purity" (Aruna, 2006). For its part, DTD is a nearly universally conserved enzyme, responsible for the cleavage of the ester bond formed between DAAs and tRNA, leading to an error free translation process (Bhatt et al., 2016). Through a mutation in the 
existing enzyme, "nature has gained enantioselectivity by a very subtle change so that the same scaffold is used for binding opposite chiral molecules and employed in different functional contexts" (Hussain et al., 2006). Yet despite the existence of these mechanisms, a number of reports say organisms use DAAs, including a recent report on the first isolation of DAA-utilizing bacteria from deep-sea sediments (Kubota et al., 2016).

The preference for LAA is notable in the chiral-selective aminoacylation of an RNA minihelix; this preference is possibly explained by the sterically restricted direction of the interaction and its further stabilization by $\mathrm{Na}^{+}$and $\mathrm{Mg}^{2+}$ ions (Englander et al., 2015; Tamura, 2011). It has also been demonstrated that compositionally simple peptides (i.e. short, without defined sequence) confer a critical phenotypic and adaptive advantage on the RNA selfreplication that occurs irrespective of chirality. By reducing RNA dependence on inorganic counterions (e.g. high concentrations of $\mathrm{Mg}^{2+}$ ), similar peptides might have helped an early RNA polymerase ribozyme in primordial RNA replication (Tagami et al., 2017). Even in the absence of initial enantiomeric excess and any chiral polarizations other than those arising from the chiral recognition between enantiomers, the nonlinear kinetics necessary for biological homochirality, called spontaneous mirror symmetry breaking, might have emerged along with RNA polymer selection mechanisms, as mathematical models show that more efficient self-replication will transition to homochirality (Jafarpour et al., 2017; Pressman et al., 2015; Ribó et al., 2017).

\subsection{Mirrored worlds}

Last but not least, a daring idea about a transition from a geochemical to a biochemical world that might have originated in two mirrored forms has been put forward (Ageno, 1972). Proteins composed entirely of DAAs and the achiral amino acid glycine are mirror images of their native counterparts composed of LAAs. Studies of interactions between antimicrobial 
proteins and achiral lipid bilayer membranes find that the bactericidal activity of $(L)$ - and $(D)$ forms of various proteins depends on whether they need to directly react with the lipid membrane to disrupt it (Zhao and Lu, 2014).

Even the mirror image, cross-chiral replicating RNA molecules may have emerged together so that both enantiomers were utilized (Sczepanski and Joyce, 2014), until LAAs were selected in the primitive aminoacylation system (based on the stereochemistry of RNA), thus enforcing the homochirality of the peptide products (Tamura, 2011, 2008). Furthermore, a prebiotically plausible mixture of natural $D$-carbohydrates may have led to the enantioenrichment of natural LAA precursors, a suggestion that stresses the importance of the "synergy between carbohydrates and amino acids in processes for enantioenrichment in both classes of molecules" (recent results and review available in Wagner et al. 2017).

However, although racemization equalizes the concentration of enantiomers, while raising the system's entropy and lowering its free energy, through polymerization, any chiral system possessing free energy forms a new, higher structural level with the same type of symmetry but with an opposite sign of chirality (Malyshko and Tverdislov, 2016). It is striking that the $\alpha$-helix secondary protein structure is right handed, as helices formed by natural amino acids and nucleotides are also predominantly right handed. The concentration and distribution of $\mathrm{Na}^{+}, \mathrm{K}^{+}$, and $\mathrm{Mg}^{2+}$ ions, along with water and pressure, are crucial for the stability and predominant handedness of nucleic acids (Pan et al., 2014). Even though they are made of asymmetric molecules, however, most homomeric protein complexes assemble at the quaternary level into energetically stable, rotationally symmetric structures (Bergendahl and Marsh, 2017; Plaxco and Gross, 2009). 


\subsection{Homochirality scenarios}

The origin of homochirality in the structure of living matter has not been fully elucidated, but 'scientists are now 'spoilt for choice' amongst possible explanations for how one enantiomer came to dominate over the other in biological molecules" (Blackmond, 2010; Blackmond and Klussmann, 2007). As we list them, homochirality origins may be abiotic, propagated due to persistence and amplification under specific conditions which were a result of abiotic circumstances. Or a statistical fluctuation could have determined the winner randomly. Or perhaps one of the enantiomers might have been more stable in terms of energy conservation, thus manifesting physical variance as a result of mirror-symmetry breaking already at the level of the physical properties of the individual molecule. If the latter is true, the chemical consequences of SB may have occurred even earlier than usually thought, at the level of the individual molecular structure, before the amplification of symmetry breaking through the aggregation of homochiral molecules.

The fact that enantiomeric excess (ee) can be directed by experimental conditions sheds new light on a long debate on which conditions and/or processes could have led to the origin of homochiral protocell and which organic molecules were the first to break the symmetry. While highlighting various abiotic mechanisms for enantioenrichment, a comprehensive review by Blackmond (2010) points out that "the burden of chiral selectivity might have been shared [between abiotic and biotic factors] as complexity increased". Such a notion leaves the "open possibility that the prebiotic molecular pool need not have evolved completely to single chirality before the formation of the first biopolymer chains" so that "the origin of biological homochirality is [not] a separate and disjoint event" (Ribó et al., 2017).

In line with this possibility, instead of simply accepting the causality principle whereby an effect (formation of enantiomerically pure polymers) cannot occur before its differential cause 
(starting from the enantiomerically pure mixture of monomers), we should also consider whether, at the critical point, SB exhibits some new global properties expressed by order parameters which emerge over time as constraints for further open-ended SB (Karunakaran et al., 2019). The most prominent example in physics is the spontaneous organization (selfassembling) of individual building blocks into ordered structures. The structures arise from particles' anisotropic shape and local order in the fluid. In contrast, the thermodynamic behavior of hard particles can be understood through entropy maximization (Damasceno et al., 2012).

As mentioned above, the "revised information theory" associates entropy, similarity (and symmetry as a measure of indistinguishability) and information (related to distinguishability) and predicts that "the evolution of the universe in general and evolution of life in particular can be quantitatively considered as a series of symmetry breaking processes" (Lin, 2001). It should be emphasized that the concept distinguishes between static and dynamic symmetry over the set of all possible microstates (i.e. a macrostructure, see Equation 5) to define SB (see Figure 5), a distinction that agrees with Montévil's more general concept of symmetry changes (2016) ${ }^{9}$. The "transference of spatial information on highly enantiopure polymers as a critical condition to support the dynamics in a self-organized biogenic system" has also been recently conceptually addressed (Cruz-Rosas et al., 2017). Thus, we might even arrive at a framework, according to which confinements or constraints could produce distinguishability (and information) for a specific property through the "unidirectional route of information transfer" (Koonin, 2015). ${ }^{10}$

\footnotetext{
${ }^{9}$ See also Footnote 4.

${ }^{10}$ It should be noted that symmetries may not be apparent in biology before they are broken, in the same fashion in which they are apparent in physics, due to the complexity of the life cycle.
} 


\section{Symmetry breaking across the life cycle}

\subsection{The structure of the cycle of SB in the biosphere}

More detailed experimental and theoretical insights into the origins and propagation of homochirality are obviously necessary. Yet irrespective of the exact origin and mechanism of propagation, we can sketch the general features of this basic layer of the biosphere.

First, we can identify and analyze symmetry breakings (asymmetries) in biology functionally, i.e. synchronously and diachronically. These layers of the biosphere are singled out by relevant SB examples. Based on the scale at which they occur, the identified cases of SB can be classified in the following way:

1. molecular: homochirality;

2. sub-cellular: cytoskeleton assembly, ion gradients across the membranes;

3. cellular: epithelial (apical-basal polarity), planar cell polarity, polarity of growth;

4. tissue-related: actin filamentation, gastrulation (emergence of diploblasts $v s$. triploblasts);

5. organism-related: left-right symmetry, inward/outward flow of matter and energy;

6. species- and higher taxa-related: evolutionary radiations and extinctions. ${ }^{11}$

Second, as explained above for the case of homochirality, a larger-scale SB can be a result of SB at a smaller scale. In fact, transitional mechanisms have been accounted for to a considerable detail across levels ( $\mathrm{Li}$ and Bowerman, 2010). To outline some of the key findings, SB at the sub-cellular level leads to persistence in the growth of cells of a certain shape (Dworkin and Losick, 2001; Guberman et al., 2008). In addition, asymmetries related to mechanisms of microtubules and actin - i.e. their structural polarity - play a key role in the initial SB and thus determine the extent and persistence of the polarization of cells, as well as

\footnotetext{
${ }^{11}$ The Central Dogma of molecular biology points out the key SB feature of the evolution of life.
} 
the polarized physical structure required for the key functions and processes of division and fusion (Irazoqui et al., 2003; Li and Gundersen, 2008; Wedlich-Soldner et al., 2003; Witte et al., 2008) ${ }^{12}$. Similarly, polarity of cytoskeletal filaments produces SB at the cellular level, streamlining the ways in which organelles and other molecular assemblies are transported (Iwasa and Mullins, 2007; Mullins, 2010; Yam et al., 2007) ${ }^{13}$. Finally, cell polarity will determine asymmetries at the level of the organism. The left-right vertebrate body asymmetries emerge due to the spatial properties of the cellular cilia (their composition, location, and orientation) that direct extracellular fluid (Aw and Levin, 2009; Hirokawa et al., 2009; Li and Bowerman, 2010; Tabin and Vogan, 2003; Vogan and Tabin, 1999). To sum up, research results over the last two decades have demonstrated how cell polarity, originating from SB at the levels of cell filaments, enables or streamlines cell motility, growth, shape, and left-right asymmetry.

All these SB mechanisms and transitions across levels are complex and require more detailed research. Each SB layer across levels of living systems has a different history, with unique details to be reconstructed in terms of its origin, stabilizing, and propagation. The plethora of possibilities places a major constraint on the determination of possible evolutionary trajectories. Yet based on what we already know about these cases, their stories are bound to be structurally similar. All face dilemmas similar to those of homochirality, irrespective of the details of their origin.

More specifically, a general structure of the emergence of SB in biological systems can be expressed as the following: SB at one level seems to persist, propagate, and result in further

\footnotetext{
${ }^{12} \mathrm{Li}$ and Gundersen (2008) provide a review of mechanisms that underlie cell polarity via asymmetries, as well as a list of relevant references.

${ }^{13}$ See Mullins (2010) for a list of relevant references.
} 
SB at different scales by becoming a stable constraint (as defined previously). The three basic aspects and stages of SB propagation, then, can be characterized as:

1. Persistence / Plasticity ${ }^{14}$

2. Accumulation / Amplification

3. Emergence of upper-scale SB

We can set this structure within the general theoretical framework outlined in previous sections. As already explained, SBs are essentially phase changes at critical points in an open system (see Figure 6), and systems characterized by such SB will exhibit robustness. This will lead to the propagation of SB in the system in the form of a particular constraint, and, typically, an SB propagation that persists long enough will eventually become a layer in the web of cascading propagations of new SBs. For example, the molecules of a certain chirality that has accumulated over time and space will become the building blocks of every new stage of $\mathrm{SB}$, as their accumulation and thus their amplification of a given macrostructure will lead to new SBs on other scales. Moreover, such emergent "assemblies" characterized by SB are plastic: they can potentially accumulate in various ways and lead to various novel SBs because they are essentially open systems (again, as characterized in detail in previous sections). Thus, in the case of living cells and multi-cellular organisms, the cascading levels start with the emergence of homochirality, continue with cytosceletal polarities, pass through tissue asymmetries, and go all the way to organismic asymmetries and radiation of taxa.

${ }^{14}$ Plasticity includes preserving asymmetry through different structures and mechanisms (Li and Bowerman, 2010, p. 3). 


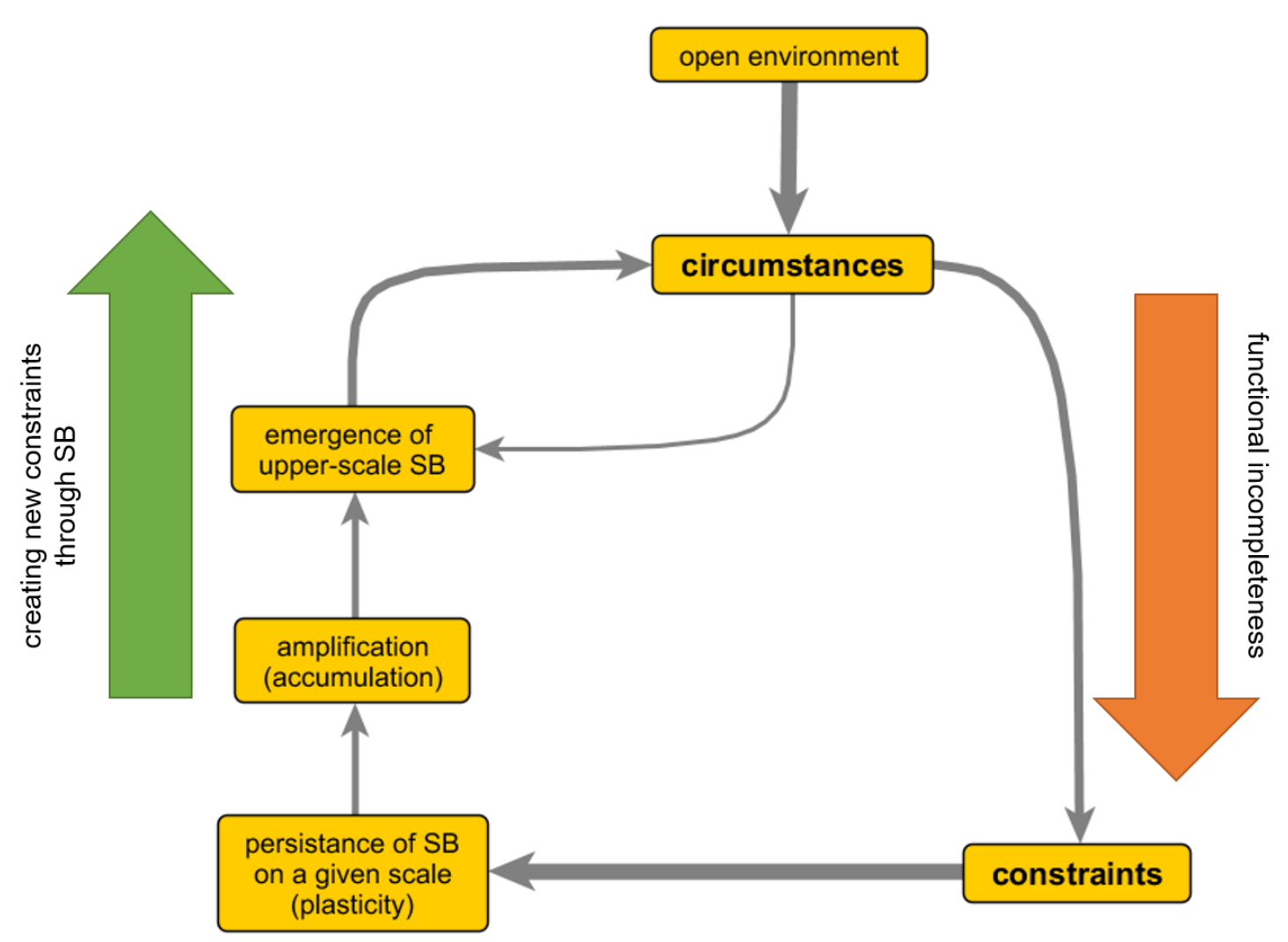

Figure 6. Life-cycle of the biosphere. The big loop flows from circumstances to constraints following the persistence and amplification of SB on one level and the emergence of new SB on upper scales. The small loop represents a dynamic system (with a chaotic attractor) that is locally unstable yet globally stable - such a circumstance can become a constraint (i.e. SB) but can also dictate how SB will cascade on upper levels.

Now, we should bear in mind that this web of cascading SB expands in a space virtually free of constraints, given the plasticity of the web. The circumstances in which SB expands eventually assume the role of constraints once a new SB layer is formed, as represented by the small loop in Figure 6. We have specified the notion of functional incompleteness as a combination of three interrelated aspects (Section 2.3), but simply summed up, given that intuitively, completeness implies the absence of any "gaps" in a specific property (which is equal to a function in the case of living organisms), a web of cascading SB is thus functionally incomplete as it exhibits a resulting "unidirectional route of information transfer" (Koonin, 2015) spanning all the levels.

Yet it is clear, based on the diagram in Figure 6, that if, for the purposes of better understanding, we set aside the specific nature of gaps in the functional space of biological 
properties as defined by the criteria provided in Section 2.3 and discussed there in detail, the incompleteness of the life cycle is apparent on a more abstract level because of three interrelated global conditions. The first is that such a life cycle is open to abiotic environmental circumstances that can eventually assume the role of constraints. The second, and perhaps the most important one (also pointed out in Section 2.3), is that this global life cycle is plastic because it is propagating itself in a virtuous circle (represented by the big loop in Figure 6) through which it is continuously regenerated, irrespective of the underlying details of how this occurs or could alternatively occur (various SB phenomena, mechanisms and routes could provide this same global property). It should be noted that such an idea is substantially similar to the general idea of extended criticality. The third and final condition is, again, that such a cycle is functionally incomplete because of the resulting "unidirectional route of information transfer" (Koonin, 2015) spanning all the levels.

To illustrate all this with an example, cytoskeletal mechanisms accumulate in such a way as to produce a very specific SB at the upper level (Mullins, 2010): e.g. the case of polarity of the cell resulting in left-right asymmetry, or the case of cilia in ciliate protozoa that can gather food only in a specific way, flapping their cilia from left to right to get food into the pouch, etc. Both external and internal factors play a role in this emergence of constraints, including the structure of the wider biochemical environment, stochasticity, and multiple feedback loops (Mullins, 2010). The entire biosphere, then, is an extended criticality cycle, a dynamic web of intertwined SB phase changes that continuously propagate.

\subsection{Conjecture: Continuity of symmetry breaking from microphysical}

\section{to the living}

SBs in biology may be just an extension, albeit a complex one, of the entire cycle that started at the microphysical scale in the cosmological past. The same elements in the early 
part of the cycle are obtained for all scales - the cycle merely became enriched at later stages with the accumulation of SBs. As the process started at a microphysical level, there were no aggregates of the entities in the Universe. Thus, some central features of the cycle, like amplification or the turning of circumstances into constraints, may not have played a role in "early" universe physics (e.g. in the plasma stage after the Big Bang) before the emergence of life, whenever it emerged, because they simply occurred too early in the process. With time, the propagation and cascading of $\mathrm{SB}$ become more pronounced, but they were either peripheral or nonexistent in the early stages of evolution. Yet given the atomic stability that derives from the selection of particular symmetry groups, SBs and their persistence in biology are essentially on a continuum. In fact, early SB played a role in the formation of life, as it enabled low-energy physical laws to emerge. These laws, in turn, enabled the emergence of biological complexity. ${ }^{15}$ Moreover, as discussed above, the parity violations within more general astrophysical conditions may have been responsible for the emergence of homochirality as the basic block of life on Earth.

Many authors have noticed a specific feature of all modern cosmological models, including the post-1998 "New standard cosmology", namely that the apparent complexity of matter has dramatically increased since the early universe by as much as 120 orders of magnitude or more (e.g. Penrose 1989; Treumann 1993; Gough 2008). It is accepted that SB is the source of this increasing complexity, although the details remain highly controversial (Ćirković, 2002; Tegmark, 1996). This could be thought of as one manifestation of the universe obtaining more and more structure with the passage of cosmic time. Since this increase is

\footnotetext{
${ }^{15}$ The more we learn about truly fundamental physics (i.e. physics happening at high energies in the very early universe), the more we see anthropic reasoning as paramount to the existence and persistence of any biological structures (see, for instance, the review by Barnes 2012).
} 
obviously extremely spatially inhomogeneous $(99.99 \ldots \%$ of the spatial volume of the presentday universe is low-complexity intergalactic space), we are entirely justified in searching for local peaks of complexity which correspond, to the best of our empirical knowledge, to habitable planets like Earth and their biospheres. Such a conclusion poses a new challenge to Copernicanism; according to complexity metrics, our location is not random or even typical any more. Finally, and most pertinently from the point of view of this paper, the natural embedding of the biological SB narrative into a wider cosmological picture points to the ultimate origin of biological information.

\subsection{Adaptation and symmetry breaking}

What sorts of questions does the analysis of life in terms of SB answer? What does it tell us about life compared, for example, to evolutionary analysis? Can we expect such an analysis to provide a general but precise account, with adaptation as one of the factors?

The amplification of SB by accumulation across scales and the production of another SB at an upper level as a result, within the circumstances that eventually turn into steady constraints, is bound to act as a major vehicle of adaptation. This requires a more general understanding of adaptation than John Maynard Smith's molecular-level one. Such an understanding should establish continuity between SB propagation and procreation.

How the initial stages of SB leading to the cycle of life occurred affects how adaptive traits should be qualified. Ageno (1972) proposed that life might have originally existed in two forms, based on either $L$ or $D$ form of amino acids. He attributed the eventual domination of the $L$ form to evolution through natural selection and competition. Other authors have suggested that codons on the genome display a preference for binding to $L$-amino acids, while certain bioinformatics studies show that the genetic code is correlated with the production of $D$-carbohydrates and $L$-amino acids. 
On the macroevolutionary level, SBs and associated phase transitions seem to have played a key role in the history of life since the Cambrian Explosion. Successive changes of macroevolutionary regimes, faunal overturns, and dramatic episodes of mass extinction have constituted the constraints relevant for all our insights into the history of life (Jablonski, 1986). Although the analogy of biological morphospace to the phase space of either classical or quantum physics has not been developed in sufficient detail, it seems adequate for the purposes of establishing a general argument. In the same manner as phase transitions represent sudden and dramatic changes in both the macroscopic properties of the system and the form of the relationship between its constituents, the analogous transitions between macroevolutionary regimes represent both changes in the global properties of the terrestrial biosphere and the switch in the ecological relationships between the constituents (populations of organisms). Parts of the biological morphospace become empty and even inaccessible, while other parts are suddenly opened. ${ }^{16}$ These transitions, most clearly associated with mass extinctions and the Cambrian radiation, propagate along trajectories set by ecological relationships, structurally akin to the phenomena at lower scales (e.g. cascading along food chains, forming local "bubbles" and "enclaves", multiple scales of SB, evolutionary innovations, etc.). The analogy with the classical phase space is strengthened by the recent realization that evolutionary convergence is ubiquitous for several reasons: observationselection effects (Vermeij, 2006), the finite size of viable genomic space (Dryden et al., 2008; McLeish, 2015), and the underlying information structure (Davies and Walker, 2016). All these developments point in the direction of more stable morphological space whose structure

16 On stability of morphospace of evolution see (Montévil 2018). For a wider discussion of convergente evolution see the issue of Interface Focus journal 2015, 5(6):20150039. 
and symmetries are firmly determined by the fundamental principles of physics, ecology, and economy.

We should emphasize that the SB feature of mass extinctions is poignant, irrespective of the exact physical causal agents of these events. Many hypotheses have been offered, but despite a few better understood exceptions, like the $\mathrm{K} / \mathrm{Pg}$ mass extinction $65 \mathrm{Myr}$ ago, our knowledge of physical and ecological causes remains sorely limited. Yet it is clear that both the causes and the impact of these episodes are predicated on our biosphere being an open system. ${ }^{17}$ Speculations about biospheric selection, based on differential survival rates (Doolittle 2014; Janković and Ćirković 2016) offer a way of joining the observed features in the history of life with the wider issues of theoretical astrobiology. Thus, the inferred features of SB on this largest macroevolutionary scales are potentially observable and empirically testable in the course of the forthcoming atrobiological research.

\section{Conclusion}

The fundamental biological scale, as a set of properties emerging through SB that remain stable trough continuous regeneration, is not the organism but the biosphere. An organism is a local biological system that can be characterized by its organizational closure (Montévil et al., 2016), or by the mutual dependency of its constraints in interaction with its environment. In contrast, first, there is an historical contingency of the biosphere (Gould and Vrba, 1982), as a

\footnotetext{
17 They have also played crucial roles in the evolution of intelligent observers. The second point is important because it tallies with the recent attempts (in many fields, from cosmology to the origin of life research) to break the hold of the artificial "Archimedean" perspective in which properties of observers are strictly separated from properties of the physical universe; the need to account for evolving sufficient functional complexity is manifest in evolutionary biology.
} 
result of the Darwinian principle of descent with modifications. ${ }^{18}$ Following our view, this principle has a functional meaning: the biosphere is essentially a thermodynamic system regenerating itself, in a virtuous thermodynamic circle. Without diminishing the importance of the stochastic changes in inheritance (namely drift, i.e. neutral theory of molecular evolution), these variations merely dovetail in this continuous regeneration. Second, there is a continuous asymmetric change in every ontogenic trajectory, even if it is counterbalanced at the local level by mutual dependency between constraints. For example, mitosis always yields different proteome distributions or differences in DNA transcriptions (Montévil et al., 2016), RNA translations, or protein expressions, so that symmetries are never preserved. ${ }^{19}$

What emerges is a general picture that should be considered, an interface of Darwinism and SB-based system analysis. The Darwinist approach is advantageous in defining the turning points of circumstances into constraints, while SB-based analysis identifies the features of a wider cycle. The latter theory tells us how to put together, in general, seemingly different processes; i.e. it identifies the structural traits of life and the biosphere. Life is not simply a thermostat controlled by positive and negative feedback (Lovelock and Margulis, 1974); it is a peculiarly complex process, controlled, on the one hand, by positive regulation from a critically extended set of constraints (continuously creating new constraints through new SB), and, on the other hand, by negative regulation like natural selection, arising from the irreversibility of the functional incompleteness the cascade creates. The complex shape of

\footnotetext{
${ }^{18}$ In fact, the contingency arises as an interplay of "three tiers": the Modern-Synthetic view of natural selection, the structural (formalist) constraints, and a couple of additional processes like the lateral gene transfer.

${ }^{19}$ We suspect that proliferation with variation is also a rule at the ontogenic level, so that if an organism does not follow this rule, it is a local biological system, not a global one. Such an assumption is accords with Soto and Sonnenschein's theory of cancer (Longo et al., 2015).
} 
organization in the biosphere can be characterized as a result of such a process, i.e., the association of two antagonistic processes, through which the biosphere evolves.

\section{Acknowledgements}

This paper was funded through grants ON 176021, ON 179048 and ON 179067.

\section{References}

Ageno, M., 1972. On molecular asymmetry in living organisms. J. Theor. Biol. 37, 187-192. https://doi.org/http://dx.doi.org/10.1016/0022-5193(72)90125-7

Aruna, R., 2006. How did protein amino acids get left-handed while sugars got right-handed? University of Illinois at Urbana-Champaign.

Aw, S., Levin, M., 2009. Is left-right asymmetry a form of planar cell polarity? Development 136, 355-366. https://doi.org/10.1242/dev.015974

Balian, 1998. Mathématiques et sciences de la nature, La jaune et la rouge. Hermann.

Barnes, L.A., 2012. The Fine-Tuning of the Universe for Intelligent Life. Publ. Astron. Soc. Aust. 29, 529-564. https://doi.org/DOI: 10.1071/AS12015

Bergendahl, L.T., Marsh, J.A., 2017. Functional determinants of protein assembly into homomeric complexes. Sci. Rep. 7, 4932. https://doi.org/10.1038/s41598-017-05084-8

Bhatt, T.K., Soni, R., Sharma, D., 2016. Recent Updates on DTD (D-Tyr-tRNA(Tyr) Deacylase): An Enzyme Essential for Fidelity and Quality of Protein Synthesis. Front. Cell Dev. Biol. 4, 32. https://doi.org/10.3389/fcell.2016.00032

Blackmond, D.G., 2010. The Origin of Biological Homochirality. Cold Spring Harb. Perspect. Biol. 2, a002147. https://doi.org/10.1101/cshperspect.a002147 
Blackmond, D.G., Klussmann, M., 2007. Spoilt for choice: assessing phase behavior models for the evolution of homochirality. Chem. Commun. 3990-3996. https://doi.org/10.1039/B709314B

Callender, C., 2001. Taking Thermodynamics Too Seriously. Stud. Hist. Philos. Sci. Part B Stud. Hist. Philos. Mod. Phys. 32, 539-553. https://doi.org/https://doi.org/10.1016/S1355-2198(01)00025-9

Ćirković, M.M., 2002. Is the Universe Really That Simple? Found. Phys. 32, 1141-1157. https://doi.org/10.1023/A:1016538827462

Cleland, C.E., 2012. Life without definitions. Synthese 185, 125-144. https://doi.org/10.1007/s11229-011-9879-7

Cleland, C.E., Chyba, C.F., 2002. Defining 'Life.' Orig. life Evol. Biosph. 32, 387-393. https://doi.org/10.1023/A:1020503324273

Collet, P., Eckmann, J.P., 2009. Iterated Maps on the Interval as Dynamical Systems, Springer Science \& Business Media. Wiley-Blackwell. https://doi.org/10.1007/978-0$8176-4927-2$

Cruz-Rosas, H.I., Riquelme, F., Maldonado, M., Cocho, G., 2017. Critical role of spatial information from chiral-asymmetric peptides in the earliest occurrence of life. Int. J. Astrobiol. 16, 28-39. https://doi.org/10.1017/S147355041500049X

Damasceno, P.F., Engel, M., Glotzer, S.C., 2012. Predictive Self-Assembly of Polyhedra into $\begin{array}{lllll}\text { Complex } & \text { Structures. } & \text { Science } \quad(80-. \quad & \text { ). }\end{array}$ https://doi.org/10.1126/science.1220869

Davies, P.C.W., Walker, S.I., 2016. The hidden simplicity of biology. Reports Prog. Phys. 79, 102601. https://doi.org/10.1088/0034-4885/79/10/102601 
De Marcellus, P., Meinert, C., Nuevo, M., Filippi, J.J., Danger, G., Deboffle, D., Nahon, L., Le Sergeant D’Hendecourt, L., Meierhenrich, U.J., 2011. Non-racemic amino acid production by ultraviolet irradiation of achiral interstellar ice analogs with circularly polarized light. Astrophys. J. Lett. 727, L27. https://doi.org/10.1088/2041$8205 / 727 / 2 / \mathrm{L} 27$

Dreiling, J.M., Gay, T.J., 2014. Chirally Sensitive Electron-Induced Molecular Breakup and the Vester-Ulbricht Hypothesis. Phys. Rev. Lett. 113, 118103. https://doi.org/10.1103/PhysRevLett.113.118103

Dryden, D.T.F., Thomson, A.R., White, J.H., 2008. How much of protein sequence space has been explored by life on Earth? J. R. Soc. Interface 5, 953-956. https://doi.org/10.1098/rsif.2008.0085

Duhem, P.M.M., 1902. Les théories électriques de J. Clerk Maxwell: Étude historique et critique. A. Hermann.

Dworkin, J., Losick, R., 2001. Differential Gene Expression Governed by Chromosomal Spatial Asymmetry. Cell 107, 339-346. https://doi.org/10.1016/S0092-8674(01)00528-1

Elliott, J.P., Dawber, P.G., 1985. Symmetry in Physics, Volume 1 and 2. Macmillan.

Englander, M.T., Avins, J.L., Fleisher, R.C., Liu, B., Effraim, P.R., Wang, J., Schulten, K., Leyh, T.S., Gonzalez, R.L., Cornish, V.W., 2015. The ribosome can discriminate the chirality of amino acids within its peptidyl-transferase center. Proc. Natl. Acad. Sci. 112, 6038-6043. https://doi.org/10.1073/pnas.1424712112

Fraassen, B.C. van, 1989. Laws and Symmetry. https://doi.org/10.1093/0198248601.001.0001

Gough, M.P., 2008. Information equation of state. Entropy. https://doi.org/10.3390/entropye10030150 
Gould, S.J., Vrba, E.S., 1982. Exaptation - a Missing Term in the Science of Form. Paleobiology 1, 4-15. https://doi.org/10.1017/S0094837300004310

Guberman, J.M., Fay, A., Dworkin, J., Wingreen, N.S., Gitai, Z., 2008. PSICIC: Noise and Asymmetry in Bacterial Division Revealed by Computational Image Analysis at SubPixel Resolution. PLoS Comput. Biol. 4, e1000233. https://doi.org/10.1371/journal.pcbi.1000233

Hazen, R.M., 2006. Presidential Address to the Mineralogical Society of America: Mineral surfaces and the prebiotic selection and organization of biomolecules. Am. Mineral. 91, $1715-1729$.

Hazen, R.M., Sverjensky, D.A., 2010. Mineral Surfaces, Geochemical Complexities, and the Origins of Life. Cold Spring Harb. Perspect. Biol. 2, a002162. https://doi.org/10.1101/cshperspect.a002162

Hirokawa, N., Tanaka, Y., Okada, Y., 2009. Left-Right Determination: Involvement of Molecular Motor KIF3, Cilia, and Nodal Flow. Cold Spring Harb. Perspect. Biol. 1, a000802. https://doi.org/10.1101/cshperspect.a000802

Hitz, T., Luisi, P.L., 2003. Chiral Amplification of Oligopeptides in the Polymerization of a-Amino Acid N-Carboxyanhydrides in Water. Helv. Chim. Acta 86, 1423-1434. https://doi.org/10.1002/hlca.200390127

Hussain, T., Kruparani, S.P., Pal, B., Dock-Bregeon, A.-C., Dwivedi, S., Shekar, M.R., Sureshbabu, K., Sankaranarayanan, R., 2006. Post-transfer editing mechanism of a Daminoacyl-tRNA deacylase-like domain in threonyl-tRNA synthetase from archaea. EMBO J. 25, 4152-4162. https://doi.org/10.1038/sj.emboj.7601278

Irazoqui, J.E., Gladfelter, A.S., Lew, D.J., 2003. Scaffold-mediated symmetry breaking by Cdc42p. Nat. Cell Biol. 5, 1062-1070. https://doi.org/10.1038/ncb1068 
Iwasa, J.H., Mullins, R.D., 2007. Spatial and Temporal Relationships between Actin-Filament Nucleation, Capping, and Disassembly. Curr. Biol. 17, 395-406. https://doi.org/10.1016/j.cub.2007.02.012

Jablonski, D., 1986. Background and Mass Extinctions: The Alternation of Macroevolutionary Regimes. Science (80-. ). 231, 129-133.

Jafarpour, F., Biancalani, T., Goldenfeld, N., 2017. Noise-induced symmetry breaking far from equilibrium and the emergence of biological homochirality. Phys. Rev. E 95, 32407. https://doi.org/10.1103/PhysRevE.95.032407

Karunakaran, S.C., Cafferty, B.J., Weigert-Muñoz, A., Schuster, G.B., Hud, N. V, 2019. Spontaneous Symmetry Breaking in the Formation of Supramolecular Polymers: Implications for the Origin of Biological Homochirality. Angew. Chemie Int. Ed. 58, 1453-1457. https://doi.org/10.1002/anie.201812808

Kauffman, S.A., 2000. Investigations. Oxford University Press.

Koonin, E. V, 2015. Why the Central Dogma: on the nature of the great biological exclusion principle. Biol. Direct 10, 52. https://doi.org/10.1186/s13062-015-0084-3

Kubota, T., Kobayashi, T., Nunoura, T., Maruyama, F., Deguchi, S., 2016. Enantioselective utilization of D-amino acids by deep-sea microorganisms. Front. Microbiol. https://doi.org/10.3389/fmicb.2016.00511

Kumar, A., Capua, E., Kesharwani, M.K., Martin, J.M.L., Sitbon, E., Waldeck, D.H., Naaman, R., 2017. Chirality-induced spin polarization places symmetry constraints on biomolecular interactions. Proc. Natl. Acad. Sci. 114, 2474-2478. https://doi.org/10.1073/pnas.1611467114

Lesne, A., Riposo, J., Roger, P., Cournac, A., Mozziconacci, J., 2014. 3D genome reconstruction from chromosomal contacts. Nat. Methods 11, 1141-1143. 
https://doi.org/10.1038/nmeth.3104

Li, R., Bowerman, B., 2010. Symmetry Breaking in Biology. Cold Spring Harb. Perspect. Biol. 2, a003475. https://doi.org/10.1101/cshperspect.a003475

Li, R., Gundersen, G.G., 2008. Beyond polymer polarity: how the cytoskeleton builds a polarized cell. Nat. Rev. Mol. Cell Biol. 9, 860-873. https://doi.org/10.1038/nrm2522

Lin, S.K., 2001. The Nature of the Chemical Process. 1. Symmetry Evolution - Revised Information Theory, Similarity Principle and Ugly Symmetry. Int. J. Mol. Sci. https://doi.org/10.3390/i2010010

Longo, G., Montévil, M., 2014. From Physics to Biology by Extending Criticality and Symmetry Breakings, in: Longo, G., Montévil, M. (Eds.), Perspectives on Organisms: Biological Time, Symmetries and Singularities. Springer Berlin Heidelberg, Berlin, Heidelberg, pp. 161-185. https://doi.org/10.1007/978-3-642-35938-5_7

Longo, G., Montévil, M., 2011. Protention and retention in biological systems. Theory Biosci. 130, 107-117. https://doi.org/10.1007/s12064-010-0116-6

Longo, G., Montévil, M., Kauffman, S., 2012. No entailing laws, but enablement in the evolution of the biosphere, in: Proceedings of the 14th Annual Conference Companion on Genetic and Evolutionary Computation. ACM, pp. 1379-1392. https://doi.org/10.1145/2330784.2330946

Longo, G., Montévil, M., Sonnenschein, C., Soto, A.M., 2015. In search of principles for a Theory of Organisms. J. Biosci. 40, 955-968. https://doi.org/10.1007/s12038-015-95749

Lovelock, J.E., Margulis, L., 1974. Atmospheric homeostasis by and for the biosphere: the gaia hypothesis. Tellus 26, 2-10. https://doi.org/10.3402/tellusa.v26i1-2.9731 
Malyshko, E. V., Tverdislov, V.A., 2016. Chirality as a physical aspect of structure formation in biological macromolecular systems. J. Phys. Conf. Ser. 741, 12065. https://doi.org/10.1088/1742-6596/741/1/012065

Maris, H.J., Kadanoff, L.P., 1978. Teaching the renormalization group. Am. J. Phys. 46, 652657. https://doi.org/10.1119/1.11224

McLeish, T.C.B., 2015. Are there ergodic limits to evolution? Ergodic exploration of genome space and convergence. Interface Focus 5. https://doi.org/10.1098/rsfs.2015.0041

Meierhenrich, U.J., 2008. Amino Acids and the Asymmetry of Life. Caught in the Act of Formation, Advances in Astrobiology and Biogeophysics.

Montévil, M., Mossio, M., 2015. Biological organisation as closure of constraints. J. Theor. Biol. 372, 179-191. https://doi.org/https://doi.org/10.1016/j.jtbi.2015.02.029

Montévil, M., Mossio, M., Pocheville, A., Longo, G., 2016. Theoretical principles for biology: Variation. Prog. Biophys. Mol. Biol. 122, 36-50. https://doi.org/https://doi.org/10.1016/j.pbiomolbio.2016.08.005

Mullins, R.D., 2010. Cytoskeletal Mechanisms for Breaking Cellular Symmetry. Cold Spring Harb. Perspect. Biol. 2, a003392. https://doi.org/10.1101/cshperspect.a003392

Myrgorodska, I., Meinert, C., Hoffmann, S. V., Jones, N.C., Nahon, L., Meierhenrich, U.J., Iuliia, M., Cornelia, M., V., H.S., C., J.N., Laurent, N., J., M.U., 2017. Light on Chirality: Absolute Asymmetric Formation of Chiral Molecules Relevant in Prebiotic Evolution. Chempluschem 82, 74-87. https://doi.org/10.1002/cplu.201600214

Nicolis, G., Prigogine, I., 1989. Exploring Complexity: An Introduction, New YorkNY WH Freeman. WH Freeman \& Company. https://doi.org/10.1063/1.2810725

Nicolis, G., Prigogine, I., 1977. Self-Organization in Nonequilibrium Systems: From 
Dissipative Structures to Order through Fluctuations, John Wiley and Sons. John Wiley and Sons. https://doi.org/10.1086/410785

Pan, F., Roland, C., Sagui, C., 2014. Ion distributions around left- and right-handed DNA and RNA duplexes: A comparative study. Nucleic Acids Res. 42, 13981-13996. https://doi.org/10.1093/nar/gku1107

Penrose, R., 1989. The Emperor's New Mind: Concerning Minds and the Laws of Physics. Oxford University Press, Oxford.

Plaxco, K.W., Gross, M., 2009. Protein Complexes: The Evolution of Symmetry. Curr. Biol. 19, R25-R26. https://doi.org/https://doi.org/10.1016/j.cub.2008.11.004

Pressman, A., Blanco, C., Chen, I.A., 2015. The RNA World as a Model System to Study the Origin of Life. Curr. Biol. 25, R953-R963. https://doi.org/https://doi.org/10.1016/j.cub.2015.06.016

Ribó, J.M., Hochberg, D., Crusats, J., El-Hachemi, Z., Moyano, A., 2017. Spontaneous mirror symmetry breaking and origin of biological homochirality. J. R. Soc. Interface 14, 20170699. https://doi.org/10.1098/rsif.2017.0699

Sczepanski, J.T., Joyce, G.F., 2014. A cross-chiral RNA polymerase ribozyme. Nature 515, 440-442. https://doi.org/10.1038/nature13900

Tabin, C.J., Vogan, K.J., 2003. A two-cilia model for vertebrate left-right axis specification. Genes Dev. 17, 1-6. https://doi.org/10.1101/gad.1053803

Tagami, S., Attwater, J., Holliger, P., 2017. Simple peptides derived from the ribosomal core potentiate RNA polymerase ribozyme function. Nat. Chem. 9, 325-332. https://doi.org/10.1038/nchem.2739

Tamura, K., 2011. Molecular basis for chiral selection in RNA aminoacylation. Int. J. Mol. 
Sci. 12, 4745-4757. https://doi.org/10.3390/ijms12074745

Tamura, K., 2008. Origin of amino acid homochirality: Relationship with the RNA world and origin of tRNA aminoacylation. Biosystems 92, 91-98. https://doi.org/10.1016/j.biosystems.2007.12.005

Tegmark, M., 1996. Does the universe in fact contain almost no information? Found. Phys. Lett. 9, 25-41. https://doi.org/10.1007/BF02186207

Treumann, R.A., 1993. Evolution of the information in the Universe. Astrophys. Space Sci. 201, 135-147. https://doi.org/10.1007/BF00626983

Vermeij, G.J., 2006. Historical contingency and the purported uniqueness of evolutionary innovations. Proc. Natl. Acad. Sci. 103, 1804-1809. https://doi.org/10.1073/pnas.0508724103

Viedma, C., 2005. Chiral Symmetry Breaking During Crystallization: Complete Chiral Purity Induced by Nonlinear Autocatalysis and Recycling. Phys. Rev. Lett. 94, 65504. https://doi.org/10.1103/PhysRevLett.94.065504

Vogan, K.J., Tabin, C.J., 1999. A new spin on handed asymmetry. Nature 397, 295-298. https://doi.org/10.1038/16796

Von Bertalanffy, L., Sutherland, J.W., 1974. General systems theory: Foundations, developments, applications, IEEE Transactions on Systems, Man, and Cybernetics. IEEE.

Wagner, A.J., Zubarev, D.Y., Aspuru-Guzik, A., Blackmond, D.G., 2017. Chiral Sugars Drive Enantioenrichment in Prebiotic Amino Acid Synthesis. ACS Cent. Sci. 3, 322328. https://doi.org/10.1021/acscentsci.7b00085

Wang, Y.-X., Gong, N., Xin, Y.-F., Hao, B., Zhou, X.-J., C.Y. Pang, C., 2012. Biological 
Implications of Oxidation and Unidirectional Chiral Inversion of D-amino Acids. Curr. Drug Metab. 13, 321-331. https://doi.org/10.2174/138920012799320392

Wedlich-Soldner, R., Altschuter, S., Wu, L., Li, R., 2003. Spontaneous Cell Polarization Through Actomyosin-Based Delivery of the Cdc42 GTPase. Science (80-. ). 299, 12311235. https://doi.org/10.1126/science.1080944

Witte, H., Neukirchen, D., Bradke, F., 2008. Microtubule stabilization specifies initial neuronal polarization. J. Cell Biol. 180, 619-632. https://doi.org/10.1083/jcb.200707042

Yam, P.T., Wilson, C.A., Ji, L., Hebert, B., Barnhart, E.L., Dye, N.A., Wiseman, P.W., Danuser, G., Theriot, J.A., 2007. Actin-myosin network reorganization breaks symmetry at the cell rear to spontaneously initiate polarized cell motility. J. Cell Biol. 178, 12071221. https://doi.org/10.1083/jcb.200706012

Zhao, L., Lu, W., 2014. Mirror image proteins. Curr. Opin. Chem. Biol. 22, 56-61. https://doi.org/10.1016/j.cbpa.2014.09.019 